\title{
Photochemical formation of oxetanes derived from aromatic ketones and substituted thiophenes and selenophenes
}

\author{
Franklin Vargas ${ }^{\dagger}$ and Carlos Rivas \\ Instituto Venezolano de Investigaciones Científicas (I.V.I.C.), Centro de Química, Laboratorio de Fotoquímica Orgánica, \\ Apartado 21827, Caracas 1020-A, Venezuela
}

\begin{abstract}
The photochemical formation of oxetanes from different heterocycles have been investigated for many years in this laboratory. Thus far, only correlation between quenching effect of the heterocycles and capability of forming oxetanes have been established but no attempts have been made to elucidate from these data the different mechanistic pathways that lead from starting materials to product in various cases. In the light of kinetic as well as ionization potential information an attempt is made in this review to establish the mechanisms involved in these reactions. By means of the studies of the Stern-Volmer plots, the quenching constants of the reaction of Paternò-Büchi between substituted thiophene and selenophene with aromatic ketones and their relationship with the potentials of ionization of these heterocycles we can infer in the efficient synthesis of oxetanes.
\end{abstract}

\section{INTRODUCTION}

For already 30 years oxetanes derived from different excited ketones and a variety of five-membered heterocycles have been prepared in this laboratory $[1,2,3$, $4,5,6,7]$. This photoaddition reaction was reported by Paternò and Chieffi from 1909. They suggested the correct structure for the photoproduct, it was not until 1954 that Büchi and collaborators reinvestigated the reaction and confirmed original work of the Italian workers. The reaction has been known since 1964 as the Paternò-Büchi reaction $[8,9,10,11]$. By far the most employed ketone in these syntheses has been benzophenone and the heterocycles used as substrates for the excited ketone have been those shown in Scheme 1.
At the time this investigation started only furan $[4,5]$ was known to yield oxetanes; other five-membered heterocycles were considered inert towards this type of cycloaddition reaction [6]. By testing different substituted heterocycles and by placing the adequate substituent on heterocycles such as pyrrole [3], thiophenes and lastly selenophene the different oxetanes were gradually prepared. It was a trial and error approach. After their quenching properties were studied it was understood why some heterocycles yield oxetanes and some are inert to the reaction. This knowledge was useful to prepare these compounds in a more systematic way. Now, with all the accumulated data it is possible to visualize the different mechanistic paths that these reactions follow.

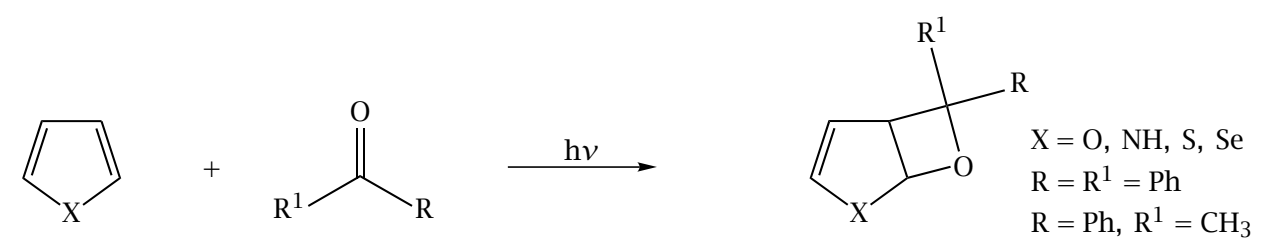

Scheme 1

The synthetic interest in the photochemical formation of oxetane extends significantly beyond its obvious value in the synthesis of oxetane containing compounds, since the photoadduct can undergo ring expansion, ring contraction or one or two bond cleavage reactions. Furthermore, it is versatile enough to allow for the introduction of substituents that could be useful in the synthesis of natural products or compounds of pharmacological interest. They have found synthetic applications since they have been used for the preparation of herbicides, fungicides, adhesives, coatings, photoresists and photoimage

\footnotetext{
${ }^{\dagger}$ E-mail: fvargas@ivic.ve
}

formulations [12, 13, 14, 15, 16, 17, 18, 19, 20, 21].

In addition to furan and methylthiophenes, unsubstituted thiophene, pyrrole, selenophene, imidazole and halogenated thiophenes have been studied as quenchers of excited benzophenone. It has also been possible to identify for the first time an oxetane derived from benzophenone and 2,5-dibromothiophene. As in previous papers of this series the reaction chosen for studying the quenching properties of the heterocycles was the photoreduction of benzophenone in isopropyl alcohol for the reasons previously stated.

Recent investigations indicate that it was only possible the formation of an unstable oxetane with 
thiophene when the benzophenone- $\mathrm{BF}_{3}$ complex is present [4]. Since this seems to be a reasonable description of the course of the reaction it is also reasonable to think that the benzophenone- $\mathrm{BF}_{3}$ complex excited by light leads to an exciplex whose excitation energy is lower than the lowest triplet energy level of thiophene which under the circumstances cannot act as a quencher and then, presumably, the electron rich heterocycle interacts with the electron deficient donor $\mathrm{BF}_{3}$ complex.

Selenophene itself does not give oxetanes in the presence of excited benzophenone. However, substitution of one methyl group in the ring suffices to make it reactive toward benzophenone upon irradiation. Thus, 2-methylselenophene, and also 3-methyl and 2,5-dimethylselenophene, under these conditions affords an oxetane in good yield [4, 22].

Besides the previous heterocycles, we have also included tellurophene in this study. This late was first tested unsuccessfully as a substrate for excited benzophenone to form an oxetane. Presumably the tellurium atom is very big and it is extruded from the ring. On the other hand, tellurophene has been used in a less common [3+4] cycloaddition as a substrate to dibromoketones thermally and photochemically in the presence of iron nonacarbonyl to yield [3.2.0] bicyclic structures conforming a seven-membered ring with the heteroatom at the bridgehead [23]. Since these compounds are very stable the idea came up to use tellurophene as a benzo[b]fused heterocycle hoping to take advantage of the additional stability expected from the aromatic ring fused to the heterocyclic nucleus. Thus, benzo[b]tellurophene was tested first with excited benzophenone. The result was the isolation of an oxetane, not isolated before, formed from the cycloaddition of the ketone across the 2,3 double bond in the heterocycle ring. On the other hand, when benzo[b]tellurophene was irradiated in the presence of benzophenone and 2,3-dimethylmaleimide the oxetane was still the only product of the reaction.

It is also interesting to note that all unsymmetrical ( $\mathrm{R}^{1}$-CO-R) carbonyl compounds that yield oxetanes give a mixture of two geometrical isomers as demonstrated by the NMR spectra of the compounds obtained. Furthermore, of the di- and trimethylthiophene derivatives used as substrates for excited benzophenone, only 2,3-dimethylthiophene afford an oxetane in fairly good yield [2]. In this case, as with the other heterocycles studied, benzophenone adds to the more substituted double bond in the ring. In the case of furan and its derivatives a detailed study of the photocycloaddition of triplet carbonyl with the heterocycle revealed a regioselectivity and especially benzaldehyde with 2,3dihydrofuran revealed also that endo-stereoselectivity is an inherent property for these processes, and it is particularly significant that only one regioisomer is formed in a diastereoisomeric ratio of $88: 12$. Increasing steric demand of the carbonyl addend leads to an increase in diastereoselectivity [24, 25, 26, 27].
The factors controlling the regio- and stereochemistry of triplet state photocycloadditions are not well understood. In contrast, the corresponding photoreactions via singlet states are more predictable, occurring with high stereospecificity. The lack of stereospecificity in the case of the triplet biradical may be explained in term of a long-lived intermediate that allows for the loss of stereospecificity before complete bonding is achieved or could be determined by the preorientation of the substrates.

A Griesbeck model explains the preferred formation of the thermodynamically less-favored endo-oxetanes derived from cyclic monoalkenes. Very recently, Griesbeck has also reported that the idea is applicable for the exo-selective formation of bicyclic oxetanes formed in the photoreaction of benzaldehyde derivatives with furan, considering the additional orbital interaction caused by the allylic radical moiety [28].

Stern-Volmer analysis of their ability to quench benzophenone photoreduction in isopropyl alcohol as well as the UV spectra of mixtures indicate that heterocycles such as pyrroles, thiophenes and selenophenes have a tendency to form exciplexes with benzophenone. It is very likely that the exciplex model could be useful to explain the regio and stereochemical course of the reaction.

\section{PATHWAYS IN THE FORMATION OF OXETANES FROM EXCESSIVE HETARENES AND BENZOPHENONE}

As reported before, thiophene and its methyl derivatives give linear Stern-Volmer plots [29, 30].

In the present investigation all the argument leading to the mechanistic pathways for oxetane formation in five-membered heterocycles will be based on the results obtained for the thiophene derivatives since in these compounds it can be found all modes of available reactions to the heterocycles studied in this laboratory. The linearity of Stern-Volmer plots is indicative that the quencher is quenching only one excited state of the benzophenone which should be its $n, \pi^{*}$ triplet state [31]. The reactivity of the methylthiophenes toward excited benzophenone has been explained previously on the basis of the argument that increasing the number of electron donating groups in the ring raises its triplet energy level to a higher value above that of the ground state relative to the unsubstituted compound. Once $T_{1}$ of the heterocycle lies above that of benzophenone energy transfer becomes inefficient and a chemical reaction takes place to form the oxetane [30]. Thiophene and its monomethylsubstituted derivatives are good quenchers and they do not form oxetanes as expected. On the other hand 2,5-dimethylthiophene is much less efficient as a quencher and forms oxetane. Furan itself as well as the methylfurans, imidazole and dicarbonylimidazole are very poor quenchers. They all form oxetanes easily. Imidazole forms an unstable oxetane, which rearranges to an alcohol function [32]. On the other hand, pyrroles and selenophenes give 
Stern-Volmer plots in the shape of a branch of a parabola. The mathematical equation for these curves:

$$
\frac{\phi_{\mathrm{o}}}{\phi}=(1+k q \tau[Q])\left(1+k q^{\prime} \tau^{\prime}[Q]\right),
$$

corresponds by $[Q]$ squared as has been verified in this laboratory by testing the data with a quadratic polynomial regression program. Where $\phi_{0}$ and $\phi$ are the quantum yields for the photoreduction of benzophenone in the absence and in the presence of quencher [Q], respectively. This result indicated that the quencher being studied was quenching two excited species.

Quenching of the photoreduction of benzophenone by thiophenes give straight line Stern-Volmer (S-V) plots which point to the fact that the increasing quenching ability of these compounds show the following sequence: 2,5-dimethyl $<$ thiophene $<3$-methyl $<2$ methyl. The worst quencher is the only one to yield an oxetane. This is a logical result, since for a very poor quencher energy transfer occurs inefficiently and the oxetane is formed.

The results obtained of investigations for the thiophene halogen derivatives show likewise that this group of compounds lies in the category of the heterocycles which give Stern-Volmer plots in the shape of a segment of a parabola. Using the same argument presented by Bolivar et al. [29] it can be shown that under the experimental conditions of this work it is not possible to quench the singlet state of benzophenone. Therefore, in addition to the triplet state the only other species that could be quenched under these conditions can presumably be an exciplex formed between the triplet state of benzophenone and a ground state molecule of the heterocycle, perhaps of the type $\left[\mathrm{H}^{+}---\mathrm{K}^{-}\right]$ (Scheme 2).

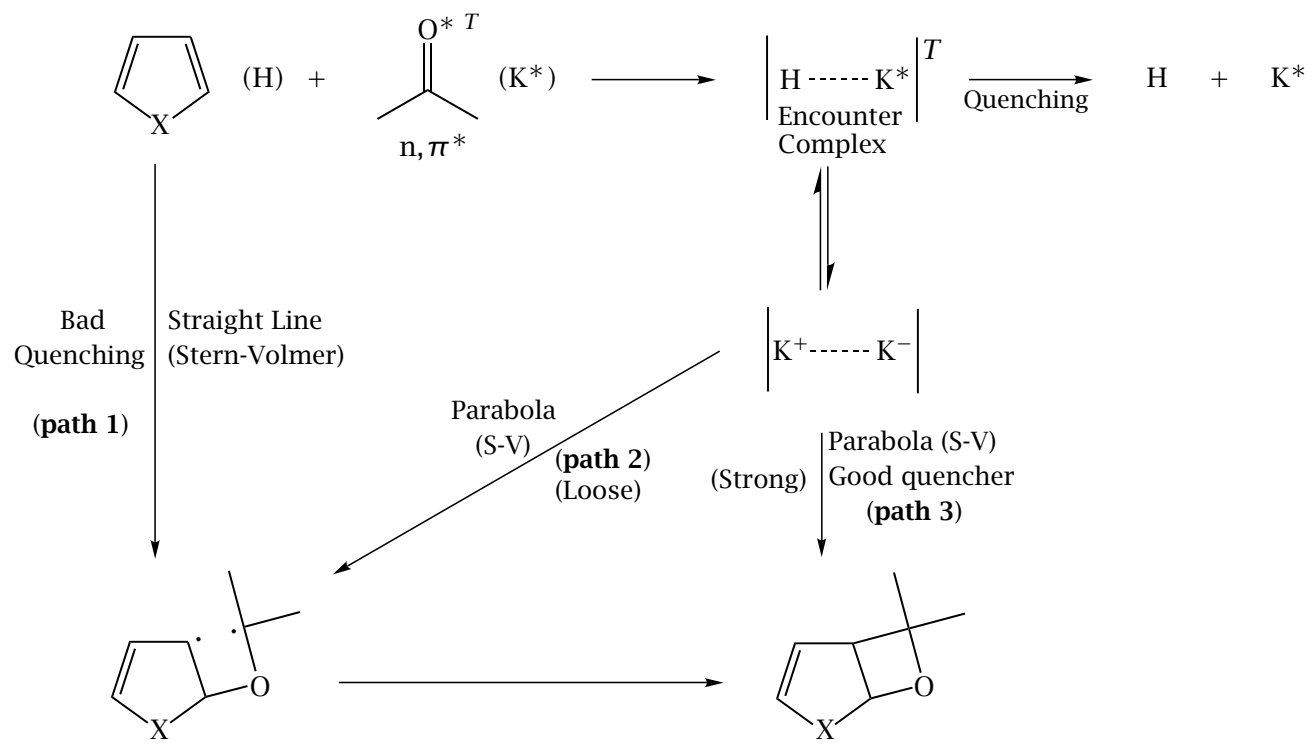

Scheme 2

The results obtained thus far indicate that the common fivemembered heterocycles may be classified in two categories, in regards to their quenching properties, those with electron donating groups such as methyl and methoxy groups, which give Stern-Volmer plots in the shape of straight lines and those substituted with halogens and electron attracting groups, such as N-benzoylpyrrole which give parabola shaped curves.

Those in the first category give oxetanes when they are bad quenchers. Since these compounds do not presumably form exciplexes they should go from starting materials to products through a biradical intermediate. On the other hand, those in the second category most likely give oxetanes when they are good quenchers, as may be shown below for 2,5-dibromothiophene, and furthermore, they go from starting materials to products through an exciplex.
Avila et al. [33] found an excellent linear correlation between quenching ability and ionization potentials of thiophene and its methyl derivatives as quenchers of the Norrish type II elimination in butyrophenone. In the methylthiophene series studied in this laboratory it was also found that a qualitative correlation between quenching ability and ionization potentials could be established. Even though there is not a good linear correlation such as that found by Avila et al. [33] a tendency in that direction may be observed. Thus, the series of methylthiophenes has been arranged in order of increasing quenching capability and the ionization potentials as well as the $k q$ 's are given under the name of each compound to illustrate what has been said. All the values of the ionization potentials were determined by electron impact technique [34]. From this information it is clear that the lower the ionization potential the better quencher the hetero- 
cycle is. However, the synthetic results and the kinetic data are a proof of the opposite behavior as for 2,5-dimethylthiophene ( $\left.8.10 \mathrm{eV}, k q=0.1 \times 10^{8} \mathrm{M}^{-1} \mathrm{~s}^{-1}\right)$ which has an even lower ionization potential and is the worse quencher of all the methylthiophenes studied. This inconsistency strengthens the explanation given previously for the reactivity of methylthiophenes [29, 30]. However, 2,5-dibromothiophene that is the best quencher and has the lowest ionization potential of all the halogen derivatives tested gives evidence of forming an oxetane. This fact and the sequence of decreasing quenching ability that may be written beginning with 2,5-dibromothiophene and ending with 3-bromothiophene suggest, that the reactivity of these compounds can be explained more adequately by correlating the stability of the above mentioned exciplexes with the ionization potentials of the heterocycles. In the halothiophenes series, the following sequence of increasing quenching ability correlates very well with decreasing ionization potentials as determined from the electron impact mass spectra technique [34]: 3-bromothiophene $(9.02 \mathrm{eV})<2$-chlorothiophene $(8.83 \mathrm{eV})<2$-bromothiophene $(8.80 \mathrm{eV})$. Another set of values of the ionization potentials obtained by photoelectron spectral techniques [34] corroborates the above argument: 2,5-dibromothiophene $(8.49 \mathrm{eV})$ $>$ 2-bromothiophene $(8.60 \mathrm{eV})>3$-bromothiophene $(8.97 \mathrm{eV})$. Values of the ionization potentials, which are slightly different from one another, may be found for the same compound depending on which methods are employed in their determination.

Therefore, if possible one should use for a series of related compounds a set of values determined by the same method. However, if values determined by different methods give a logical trend in a general direction very helpful qualitative correlation may be established, as for example in the present case, between quenching ability and ionization potentials of halothiophenes. Thus, this information may be useful for postulation of a general mechanism of oxetane formation in heterocycles. It is likely that the exciplexes are loose complexes, which become more stable as the ionization potential of the heterocycle becomes very low, as may be the case with 2,5-dibromothiophene. In this series as well as in the previous one the quenching ability increases as the ionization potential decreases. However, when the exciplex becomes very stable, as with 2,5dibromothiophene, either it may be quenched or it may collapse into an oxetane either through a biradical intermediate or directly. As an analogy to the charge transfer complex suggested by Loutfy and Yip [35] for the interaction between chloro and bromobenzenes with excited triplet benzophenone, we depict the exciplex intermediate as $\left[\mathrm{H}^{+}----\mathrm{K}^{-}\right]^{\mathrm{T}}$ because the electron rich species is the thiophene derivative while the excited carbonyl moiety is electron deficient. Therefore, electron density flows from the heterocycle to the carbonyl compound; this suggestion is corroborated by the inverse relationship between ionization potentials and $k q$ 's. The fol- lowing scheme may be useful to summarize the above (Scheme 2).

The alkyl and alkoxy thiophene series indicated the same tendencies of an increase of the quenching constants when the ionization potential diminishes [36]. For example: thiophene $(9.12 \mathrm{eV} ; k q=$ $\left.1.30 \times 10^{-8} \mathrm{M}^{-1} \mathrm{~s}^{-1}\right)$, 3-methylthiophene $(8.72 \mathrm{eV} ; k q=$ $\left.1.60 \times 10^{-8} \mathrm{M}^{-1} \mathrm{~s}^{-1}\right)$, 2-methylthiophene $(8.63 \mathrm{eV} ; \mathrm{kq}=$ $\left.3.30 \times 10^{-8} \mathrm{M}^{-1} \mathrm{~s}^{-1}\right), \quad$ 2-methoxythiophene $(8.30 \mathrm{eV}$; $\left.k q=4.40 \times 10^{-8} \mathrm{M}^{-1} \mathrm{~s}^{-1}\right)$.

Benzo[b]selenophenes show a similar behavior as bromothiophenes in the sense that their Stern-Volmer plots are not straight lines but segments of a parabola. In the selenophene series, selenophene itself does not form an oxetane while the methylselenophenes do form oxetanes when irradiated in the presence of benzophenone alone, but 2,5-dimethylselenophene can form the oxetane both in the presence of benzophenone alone and in the reaction mixture containing benzophenone and 2,3-dimethylmaleimide, in this instance no [2+2] cycloaddition product is isolated.

In the selenophene series the quenching ability increases from selenophene to 2,5-dimethylselenophene as follows: selenophene $<3$-methyl $<2$-methyl $<2$,5dimethyl. Studies carried out in this laboratory on bromothiophenes give a similar sequence as follows: thiophene $<3$-bromo $<2$-bromo $<2$,5-dibromo. In this series the only one that gives evidence of forming oxetane is 2,5-dibromothiophene.

The shape of the Stern-Volmer curves for selenophenes (parabola) suggests the presence of a charge transfer complex between the heterocycle and benzophenone.

Furthermore, it may be speculated that from all the data available it may be inferred that when SternVolmer plots are straight lines the worst quenchers yield oxetanes while when the Stern-Volmer plots are curved (segments of a parabola) the opposite takes place and the best quencher yields oxetane. The reason for this behavior may be found in the fact that the charge transfer (exciplex, in the excited state) between benzophenone and the heterocycle is held very strongly and collapses to the oxetane competing with the [2+2] cycloaddition reaction between carbon-carbon double bonds. Surprisingly enough 3,4-dimethyl-substituted heterocycles do not have a tendency to form oxetanes as has been observed for thiophenes and selenophenes.

3-Methyl and 2-methyl-selenophenone are better quenchers than selenophene and also form a charge transfer complex with the electron deficient excited benzophenone. For this same reason, perhaps, oxetanes derived from these two heterocycles are only possible when no competing dienophiles as for example maleic anhydride derivatives are present.

As a conclusion, one can safely propose that in general: a) Oxetanes derived from heterocycles which give linear Stern-Volmer plots go from starting materials to products through a biradical intermediate (path 1); this is the case of furans and dimethylthiophenes. 
b) Oxetanes derived from heterocycles, which give Stern-Volmer plots in the shape of a segment of a parabola, may follow either path 2 or 3 ; this is the case of $\mathrm{N}$-substituted pyrroles and selenophenes.

\section{REFERENCES}

[1] C. Rivas, M. Velez, and O. Crescente, Chem. Commun. (1970), 1474.

[2] C. Rivas and R. A. Bolivar, J. Heterocyclic Chem. 10 (1973), 967.

[3] C. Rivas and R. A. Bolívar, J. Heterocyclic Chem. 13 (1976), 1037.

[4] F. Vargas, C. Rivas, M. Navarro, and Y. Alvarado, J. Photochem. Photobiol. A: Chem. 93 (1996), 169.

[5] C. Rivas and F. Vargas, CRC Press, INC. 44 (1995), 536.

[6] G. R. Evanega and E. B. Whipple, Tetrahedron Lett. (1967), 2163.

[7] C. Rivas, R. A. Bolívar, and M. Cucarella, J. Heterocyclic Chem. 19 (1982), 529.

[8] E. Paterno and G. Chieffi, Gazz. Chim. Ital. 39 (1909), 341.

[9] G. Büchi, C. G. Inman, and E. S. Lipinski, J. Am. Chem. Soc. 76 (1954), 4327.

[10] N. C. Yang, Pure and Appl. Chem. 6 (1964), 591.

[11] N. C. Yang, M. Kimura, and W. Eisenhardt, J. Am. Chem. Soc. 95 (1973), 5058.

[12] A. R. Howell and A. J. Ndakala, Organic Lett. 1 (1999), 825.

[13] S. H. Rosenberg, K. P. Spina, H. Stein, J. Cohen, W. R. Baker, and H. D. Kleinert, Bioorg. Med. Chem. 9 (1994), 927.

[14] M. M. Wang, B. Cornett, J. Nettles, D. C. Liotta, and J. P. Snyder, J. Org. Chem. 65 (2000), 1059.

[15] S. Ishii, M. Zhuo, Y. Yoshida, and H. Noguchi, Synth. Commun. 29 (1999), 3207.

[16] C. A. Dvorak, C. Dufour, S. Iwasa, and V. H. Rawal, J. Org. Chem. 63 (1998), 5302.

[17] T. Bach and K. Kather, J. Org. Chem. 61 (1996), 7642.
[18] K. Ito, M. Yoshikate, and T. Katsuki, Heterocycles 42 (1996), 305.

[19] B. Guichard, V. Poirier, C. Noel, D. Reyx, A. Leborgne, M. Leblane, M. Large, and F. Kajzar, Macromol. Chem. Phys. 197 (1996), 3631.

[20] B. Das and S. P. Rao, Indian J. Chem. (B) 35 (1996), 883.

[21] N. Hurdoc, A. Stoleru, D. Pavel, and C. I. Simionescu, J. Thermal Anal. 47 (1996), 735.

[22] C. Rivas, D. Pacheco, and F. Vargas, Acta Sud. Amer. de Quim. 2 (1982) 1; Chem. Abstr. 99 (1983), 38394.

[23] D. Pacheco, F. Vargas, and C. Rivas, Phosphorud and Sulfur 25 (1985), 245.

[24] A. G. Griesbeck and S. Stadtmüller, J. Am. Chem. Soc. 113 (1991) 6923.

[25] M. Abe, E. Torii, and M. Nojima, J. Org. Chem. 65 (2000) 3426.

[26] T. Bach, K. Jodicke, K. Kather, and R. Frohlich, J. Am. Chem. Soc. 119 (1997), 2437.

[27] M. Abe, K. Fujimoto, and M. Nojima, J. Am. Chem. Soc. 122 (2000), 4005.

[28] A. G. Griesbeck, S. Buhr, M. Fiege, H. Schmickler, and J. Lex, J. Org. Chem. 63 (1998) 3847.

[29] R. A. Bolívar and C. Rivas, J. Photochem. 19 (1982), 95.

[30] R. A. Bolívar, R. Machado, L. Montero, F. Vargas, and C. Rivas, J. Photochem. 22 (1983), 91.

[31] A. Barltrop and J. D. Coyle, Excited States in Organic Chemistry, Wiley, 1975.

[32] T. Matsuura, A. Banba, and K. Ogura, Tetrahedron 27 (1971), 1211

[33] A. Avila, S. Braslavsky, and J. C. Scaiano, J. Photochem. 4 (1975), 375.

[34] G. Aloisi and S. Pignataro, J. Chem. Soc. Faraday Trans. I. 69 (1973), 534.

[35] R. O. Loutfy and R. W. Yip, Can. J. Chem. 51 (1973), 1881.

[36] Z. Bozic, K. Humski, T. Cvitas, and L. Klasinc, J. Chem. Soc. Perkin II. (1977), 1413. 


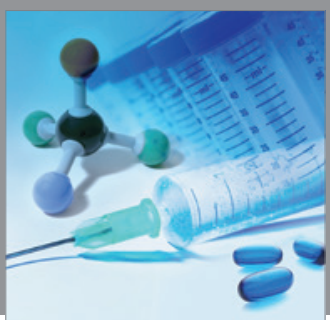

International Journal of

Medicinal Chemistry

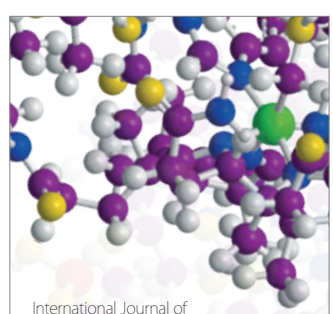

Carbohydrate Chemistry

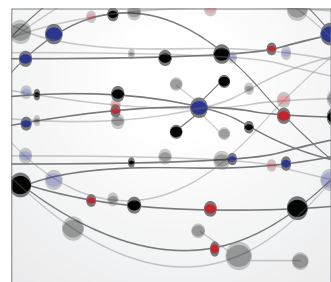

The Scientific World Journal
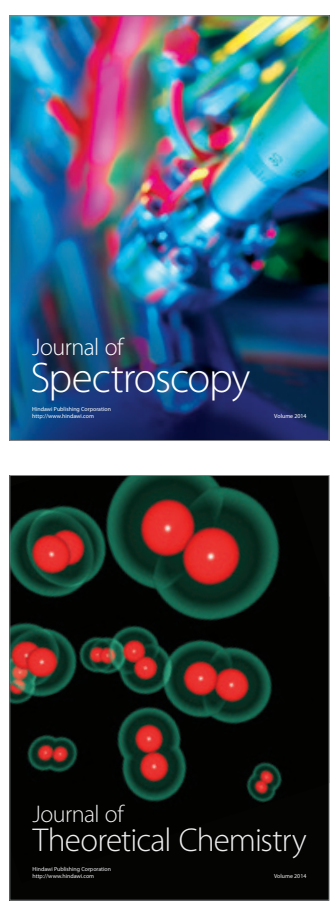
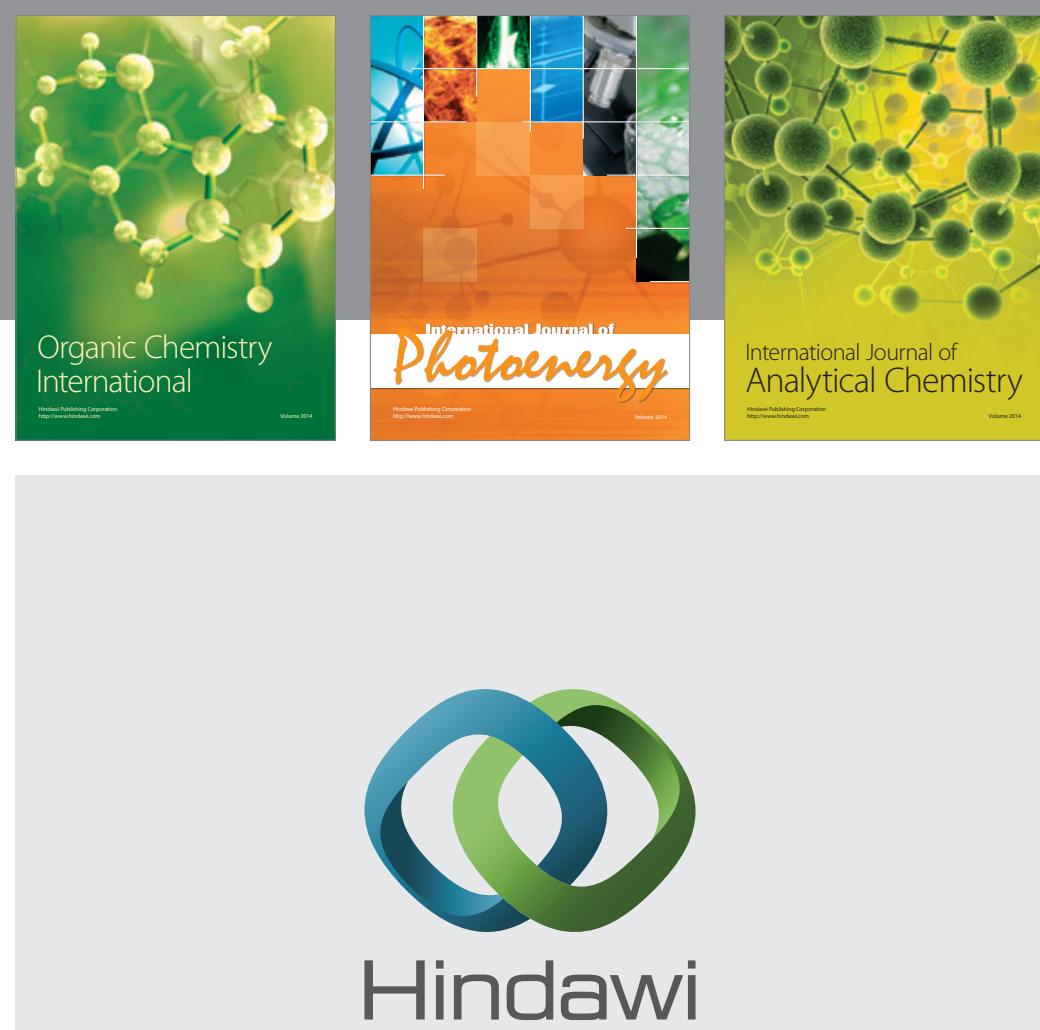

Submit your manuscripts at

http://www.hindawi.com
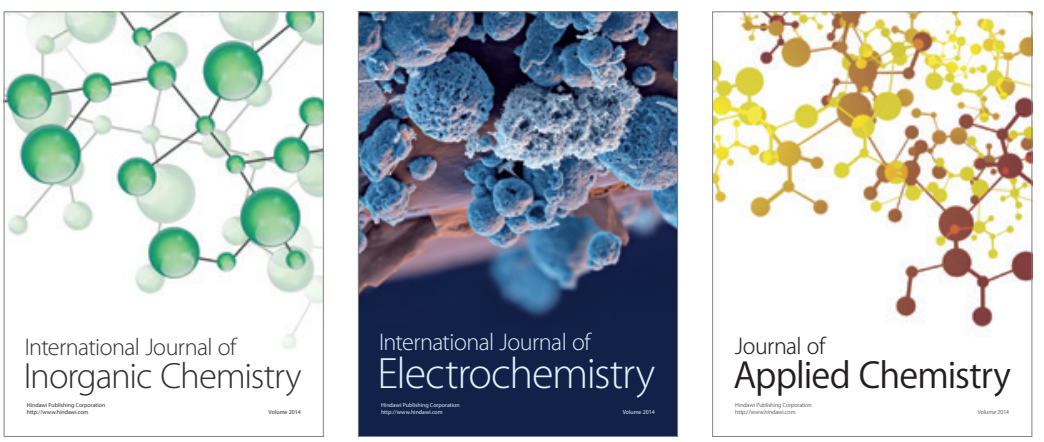

Journal of

Applied Chemistry
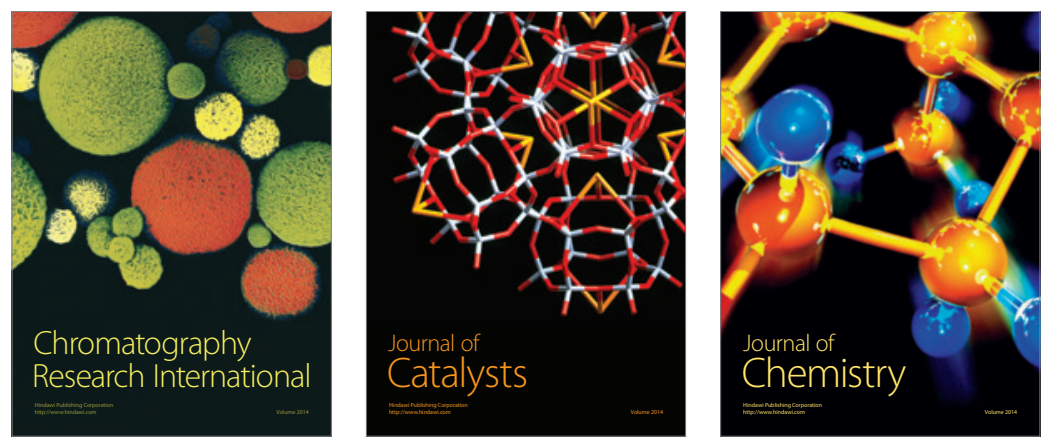
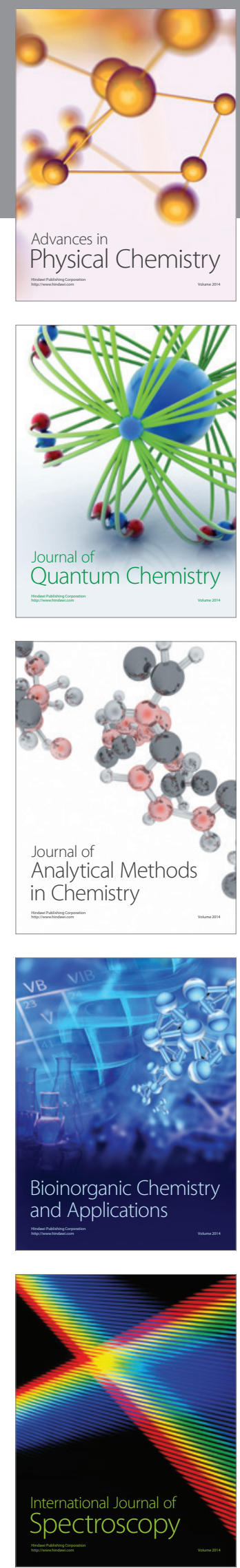